\title{
A background galaxy in the field of the $\beta$ Pictoris debris disk ${ }^{\star}$ (Research Note)
}

\author{
S. Regibo ${ }^{1}$, B. Vandenbussche ${ }^{1}$, C. Waelkens ${ }^{1}$, B. Acke ${ }^{1, \star \star}$, B. Sibthorpe ${ }^{2}$, M. Nottebaere ${ }^{1}$, K. Voet $^{1}$, \\ J. Di Francesco ${ }^{3}$, M. Fridlund ${ }^{4}$, W. K. Gear ${ }^{5}$, R. J. Ivison ${ }^{2}, 6$, and G. Olofsson ${ }^{7}$ \\ ${ }^{1}$ Instituut voor Sterrenkunde, KU Leuven, Celestijnenlaan 200D, 3001 Leuven, Belgium \\ e-mail: sara@ster.kuleuven.be \\ 2 UK Astronomy Technology Centre, Royal Observatory Edinburgh, Blackford Hill, Edinburgh EH9 3HJ, UK \\ ${ }^{3}$ National Research Council of Canada, Herzberg Institute of Astrophysics, 5071 West Saanich Road, Victoria, BC, V9E 2E7, Canada \\ ${ }^{4}$ ESA Research and Science Support Department, ESTEC/SRE-S, Keplerlaan 1, 2201 AZ Noordwijk, The Netherlands \\ 5 School of Physics and Astronomy, Cardiff University, Queens Buildings The Parade, Cardiff CF24 3AA, UK \\ ${ }^{6}$ Institute for Astronomy, University of Edinburgh, Blackford Hill, Edinburgh EH9 3HJ, UK \\ 7 Department of Astronomy, Stockholm University, AlbaNova University Center, Roslagstullbacken 21, 10691 Stockholm, Sweden
}

Received 12 December 2011 / Accepted 24 February 2012

\begin{abstract}
Herschel images in six photometric bands show the thermal emission of the debris disk surrounding $\beta$ Pic. In the three PACS bands at $70 \mu \mathrm{m}, 100 \mu \mathrm{m}$ and $160 \mu \mathrm{m}$ and in the $250 \mu \mathrm{m}$ SPIRE band, the disk is well-resolved, and additional photometry is available in the SPIRE bands at $350 \mu \mathrm{m}$ and $500 \mu \mathrm{m}$, where the disk is only marginally resolved. The SPIRE maps reveal a blob to the southwest of $\beta$ Pic, coinciding with submillimetre detection of excess emission in the disk. We investigated the nature of this blob. Our comparison of the colours, spectral energy distribution and size of the blob, the disk and the background sources shows that the blob is most likely a background source with a redshift between $z=1.0$ and $z=1.6$.
\end{abstract}

Key words. stars: early-type - stars: individual: $\beta$ Pictoris - planetary systems - techniques: image processing

\section{Introduction}

The circumstellar disk around the A6V star $\beta$ Pic was discovered by IRAS in 1983 (Aumann et al. 1984) and especially the inner part has been studied extensively ever since (Kalas \& Jewitt 1995; Pantin et al. 1997; Mouillet et al. 1997; Heap et al. 2000; Telesco et al. 2005). Optical observations imply that the disk extends to 95" offset, which corresponds to 1800 AU (Larwood \& Kalas 2001). Warps and asymmetries in the inner part of the disk suggest the presence of planetesimals and even planets, the latter of which has been confirmed by Lagrange et al. (2010).

Owing to its relative proximity to the Earth $(19.44 \pm 0.05 \mathrm{pc}$; van Leeuwen 2007), the debris disk was resolved spatially at longer wavelengths as well (Holland et al. 1998; Nilsson et al. 2009; Liseau et al. 2003).

The $\beta$ Pic debris disk has been imaged by Herschel in six photometric bands. In the $250-500 \mu \mathrm{m}$ bands a blob appears to the southwest of the star. This blob coincides with the one observed at $850 \mu \mathrm{m}$ with SCUBA by Holland et al. (1998) and $870 \mu \mathrm{m}$ with LABOCA by Nilsson et al. (2009) and both teams of authors assumed it to be a structure in the disk.

A first counter-argument was already mentioned in Dent et al. (2000). Assuming the blob indeed originates in the debris disk and has the same dust characteristics, its observed emission

* Herschel is an ESA space observatory with science instruments provided by European-led Principal Investigator consortia and with important participation from NASA.

$\star \star$ Postdoctoral Fellow of the Fund for Scientific Research, Flanders. at $850 \mu \mathrm{m}$ can only be explained by at least $1.2 M_{\text {Moon }}$, a mass comparable to the total mass of the disk in small dust particles. Given the blob's distance from the central star and the fact that there is no counterpart for such a large amount of dust in deep optical observations, Dent et al. (2000) argued that the blob cannot be a structure in the disk. Vandenbussche et al. (2010) argued that given the high amount of background galaxies in the neighbourhood of $\beta$ Pic, the blob probably is a background source.

In this paper, we investigate the hypothesis that the blob is a background source and not a structure in the circumstellar disk.

\section{Observations and data reduction}

Photometric maps were obtained with the Herschel Space Observatory (Pilbratt et al. 2010) with the PACS and SPIRE photometers. A concise observing log can be found in Table 1 . More information concerning the scientific capabilities, observing modes, data reduction, calibration, and performance of these instruments can be found in Poglitsch et al. (2010) for PACS, and in Griffin et al. (2010) and Swinyard et al. (2010) for SPIRE.

The observations were performed in the context of the "Stellar Disk Evolution" guaranteed time key programme (PI: G. Olofsson) during the science demonstration phase.

Pipeline processing as well as image analysis have entirely been made in the interactive processing environment HIPE, which has been developed for the Herschel project (Ott \& Science Ground Segment Consortium 2010). 

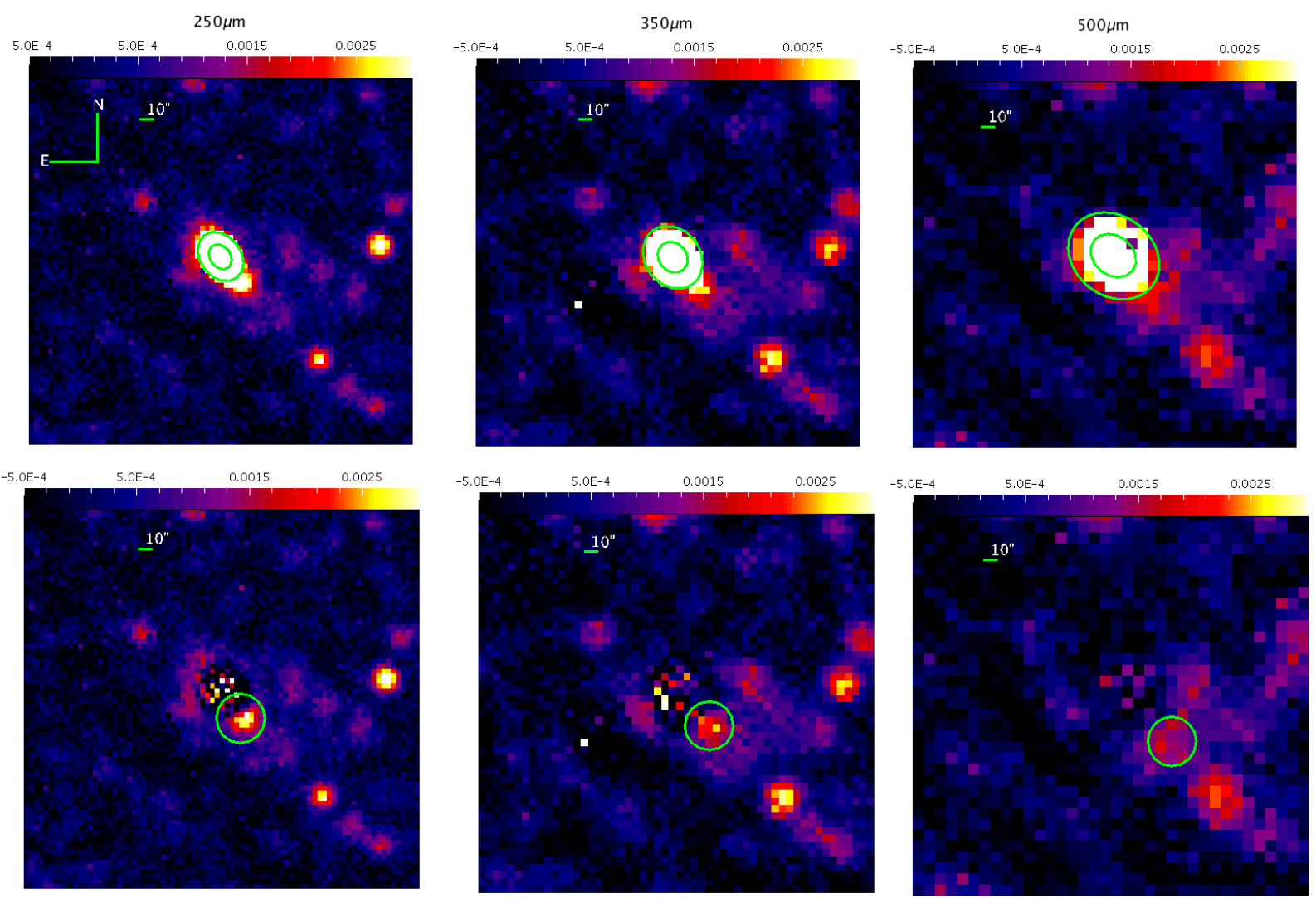

Fig. 1. Surface brightness maps in the SPIRE bands (in the upper panels) with the fitted Gaussian overplotted; and the maps after subtraction of the fitted disk, with the position of the blob indicated with a 20" radius circle in the lower panels (left: $250 \mu \mathrm{m} ;$ middle: $350 \mu \mathrm{m}$; right: $500 \mu \mathrm{m}$ ). All maps display the same area on the sky and are centred on the optical position of $\beta$ Pic.

Table 1. Observation log for the photometric Herschel observations of $\beta$ Pic.

\begin{tabular}{lcccc}
\hline \hline & Observation & Date & Duration & Filters $[\mu \mathrm{m}]$ \\
\hline SPIRE & 1342187327 & $2009-11-30$ & $3336 \mathrm{~s}$ & $250,350,500$ \\
PACS & 1342185457 & $2009-10-07$ & $808 \mathrm{~s}$ & 100,160 \\
PACS & 1342186612 & $2009-11-01$ & $5506 \mathrm{~s}$ & 70,160 \\
PACS & 1342186613 & $2009-11-01$ & $5506 \mathrm{~s}$ & 70,160 \\
\hline
\end{tabular}

Notes. Column 1 gives the instrument, Col. 2 the observation identification, Col. 3 the date of the observation, Col. 4 its duration and Col. 5 the observed wavelengths.

\subsection{SPIRE observations}

The SPIRE observation includes three wavelength bands simultaneously (at $250 \mu \mathrm{m}, 350 \mu \mathrm{m}$ and $500 \mu \mathrm{m}$ ), covering an area of $8^{\prime} \times 8^{\prime}$ homogeneously. The maps were constructed with the naiveMapper task. This algorithm projects each sample onto a single map pixel. The pixel size is $4^{\prime \prime}, 6^{\prime \prime}$ and $9^{\prime \prime}$ in the three bands respectively, and the beam FWHM is 18.2", 24.9" and $36.3^{\prime \prime}$ respectively.

\subsection{PACS observations}

At $100 \mu \mathrm{m}$, we have a single shallow PACS scan map, taken at medium speed $\left(20^{\prime \prime} \mathrm{s}^{-1}\right)$; while at $70 \mu \mathrm{m}$ and $160 \mu \mathrm{m}$, a scan and a cross-scan at low speed $\left(10^{\prime \prime} \mathrm{s}^{-1}\right)$ are at our disposal. For the shallow observation, an area of $2^{\prime} \times 2^{\prime}$ is covered homogeneously; for the deep observations an area of $2.5^{\prime} \times 2.5^{\prime}$.
The PACS observations were processed with the HIPE pipeline script for the reduction of observations of extended sources, such as $\beta$ Pic. Removal of cosmic rays was performed with the IIndLevelDeglitch task. For each pixel, $\sigma$-clipping was applied on all contributions before projecting the remaining values onto a map. The flux conversion was made with version 6 of the response calibration. Before applying a high-pass filter to eliminate low-frequency drifts, the region around the source was masked. The width of the filter window was taken as half the scan leg length (3.7"). We used the photProject task to combine the detector time series signals into maps of $1^{\prime \prime}$ pixels in the blue and green, and $2^{\prime \prime}$ pixels in the red, weighting the signal by the respective noise.

\section{Image analysis}

In this section we discuss how we determined the flux of the debris disk, the blob, and the background sources.

From the SPIRE maps shown in Fig. 1, a blob to the southwest of the debris disk of $\beta$ Pic can be clearly seen (at an offset $(\Delta \alpha, \Delta \delta)=\left(-25^{\prime \prime},-23^{\prime \prime}\right)$ from the star $)$, after subtraction of the flux of the disk. In Fig. 2, we notice tens of background sources in the $10^{\prime} \times 10^{\prime}$ region around the star. We quantified whether the far-IR colours of the blob resemble the colours of these other sources more than the colours of the debris disk itself. If so, this would be a strong indication that the blob is a background source and not a structure in the disk.

In the PACS maps, the background sources are less prominent, and the complex shape of the PACS point-spread-function (PSF) prohibits a swift separation of the disk flux from the PACS maps. Moreover, the surface brightness profiles for PACS 


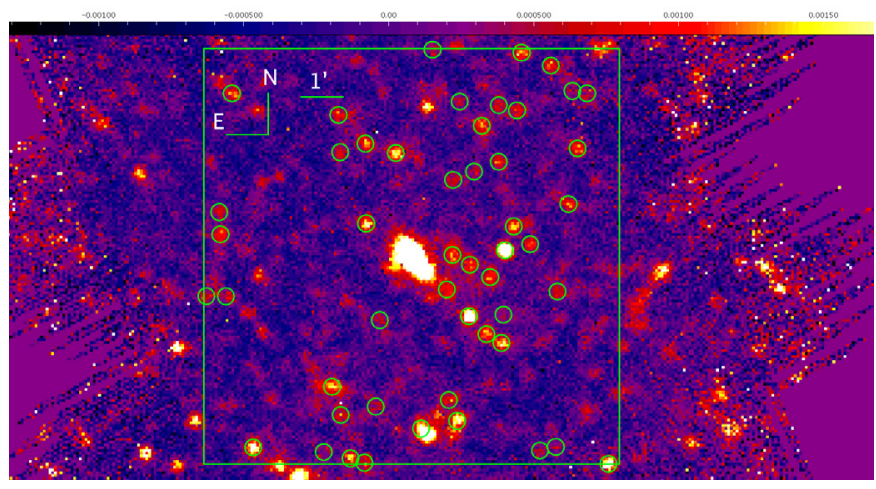

Fig. 2. SPIRE map at $250 \mu \mathrm{m}$ on which the background sources are indicated with green circles. The green rectangle indicates the region that is covered by the two scan directions.

Table 2. Fit parameters for the subtracted Gaussian disk model in the SPIRE wavelength bands.

\begin{tabular}{lccc}
\hline \hline Parameter & $250 \mu \mathrm{m}$ & $350 \mu \mathrm{m}$ & $500 \mu \mathrm{m}$ \\
\hline$\alpha_{\text {center }}[\mathrm{h} \mathrm{m} \mathrm{s}]$ & 054717.12 & 054717.11 & 054716.94 \\
$\delta_{\text {center }}\left[{ }^{\circ}{ }^{\prime \prime}\right]$ & -510355.5 & -510355.52 & -510358.20 \\
$F W H M_{\text {major axis }}\left[{ }^{\prime \prime}\right]$ & 23.20 & 28.96 & 43.01 \\
$F W H M_{\text {minor axis }}\left[{ }^{\prime \prime}\right]$ & 17.76 & 23.54 & 34.78 \\
Position angle $\left[{ }^{\circ}\right]$ & 37.40 & 39.31 & 39.31 \\
\hline
\end{tabular}

in Vandenbussche et al. (2010, see Fig. 3) suggest the blob is faint and cannot be discerned in the PACS wavelength range.

In the SPIRE maps, we separated the fluxes of the blob from the fluxes of the debris disk by fitting an elongated Gaussian to the observed disk and subtracting it, using the sourceFitting and imageSubtract tasks in HIPE. Figure 1 shows the original SPIRE maps on which the respective fitted Gaussians were overplotted, and the residual maps with the blob indicated. The fit parameters are summarised in Table 2.

We can now determine the flux of the debris disk and of the blob by performing aperture photometry on the original maps and on the difference maps with the rectangularSkyAperturePhotometry task in HIPE. This task calculates the flux within a circular aperture and estimates a sky contribution from a rectangular region close enough to the target to achieve a similar coverage.

To calculate the flux of the disk, we used a circular $60^{\prime \prime}$ radius target aperture. A value for the sky contribution was obtained with a sky estimation algorithm, corresponding to the $\mathrm{mmm}$ routine from the IDL Astronomy Library to estimate the background in a stellar contaminated field. The acquired fluxes are summarised in Table 3 . The errors on the fluxes are dominated by uncertainties in flux calibration, which are less than $15 \%$ for SPIRE (Swinyard et al. 2010), less than $10 \%$ at $70 \mu \mathrm{m}$ and $100 \mu \mathrm{m}$, and less than $20 \%$ at $160 \mu \mathrm{m}$ for PACS (Poglitsch et al. 2010). To obtain the flux of the blob, we used a target radius of $20^{\prime \prime}$ on the difference maps. The results are summarised in Table 4. In the difference maps, the residuals from the disk subtraction become substantial, especially at $250 \mu \mathrm{m}$. The errors on the fluxes of the blob are hence calculated as the standard deviation on the flux in 20 circular apertures (with a radius of $20^{\prime \prime}$ ) close to the subtracted disk.

To determine an upper limit for the flux of the blob in the PACS maps, we calculated the flux within a $10^{\prime \prime}$ aperture at the position of the blob (in the original images) and subtracted the flux within a $10^{\prime \prime}$ aperture on the opposite side of the star.
Table 3. Flux of the debris disk for PACS and SPIRE (calculated for a $60^{\prime \prime}$ target radius).

\begin{tabular}{lc}
\hline \hline Wavelength $[\mu \mathrm{m}]$ & Flux $[\mathrm{Jy}]$ \\
\hline 70 & $15.34 \pm 0.8$ \\
100 & $9.34 \pm 0.5$ \\
160 & $4.55 \pm 0.5$ \\
250 & $1.88 \pm 0.14$ \\
350 & $0.81 \pm 0.06$ \\
500 & $0.37 \pm 0.03$ \\
\hline
\end{tabular}

Table 4. Upper limits for the flux of the blob in the PACS images and the flux of the blob in the three SPIRE bands (calculated for a 20" target radius), and the corresponding beam FWHM.

\begin{tabular}{lcc}
\hline \hline Wavelength $[\mu \mathrm{m}]$ & Flux $[\mathrm{Jy}]$ & Beam $\left[{ }^{\prime \prime}\right]^{a}$ \\
\hline 70 & $<0.39$ & $5.26 \times 5.61$ \\
100 & $<0.16$ & $6.69 \times 6.89$ \\
160 & $<0.15$ & $10.46 \times 12.06$ \\
250 & $0.081 \pm 0.027$ & 18.2 \\
350 & $0.050 \pm 0.006$ & 24.9 \\
500 & $0.026 \pm 0.004$ & 36.3 \\
\hline
\end{tabular}

Notes. (a) Taken from the PACS Observer's Manual and SPIRE Observers' Manual (version 2.4).

Scaling the difference in flux between the SW- and NE-aperture with the encircled energy function yields the upper limits listed in Table 4.

To identify the background sources, we applied the HIPE sourceExtractorSussextractor task to the original map at $250 \mu \mathrm{m}$ on the homogeneously covered region (with signal-to-noise threshold of 5.0). This task yields a list of sky coordinates and fluxes for the extracted sources, which can be fed into the task as priors when applying it to the two other original maps. This forces the task to extract fluxes for the same sources in all three images. Sources within a radius of $50^{\prime \prime}$ from the star were removed from the list and 50 sources were left for analysis (see Fig. 2). Their sky positions and fluxes in the SPIRE bands are summarised in Table A.1.

\section{Discussion}

\subsection{Comparison of the far-IR colours of the blob, the disk and the background sources}

Figure 4 shows $F_{500} / F_{350}$ vs. $F_{250} / F_{350}$ colour-colour diagram for the debris disk, the blob and the background sources. The colours of the blob are more similar to the colours of the background sources than the colours of the disk. This is a first indication that the blob is a background source and not a structure in the disk, but no decisive conclusion can be drawn due to the relatively large error bars.

\subsection{Comparison of the size of the blob and the background sources}

A second argument that the blob is a background source was found in the comparison between the size of the blob and the size of the background sources. The full-width at half-maximum (FWHM) of the background sources was determined in the three SPIRE bands by fitting a Gaussian to each of the sources in the original maps. A Gaussian fit to the blob in the difference maps 

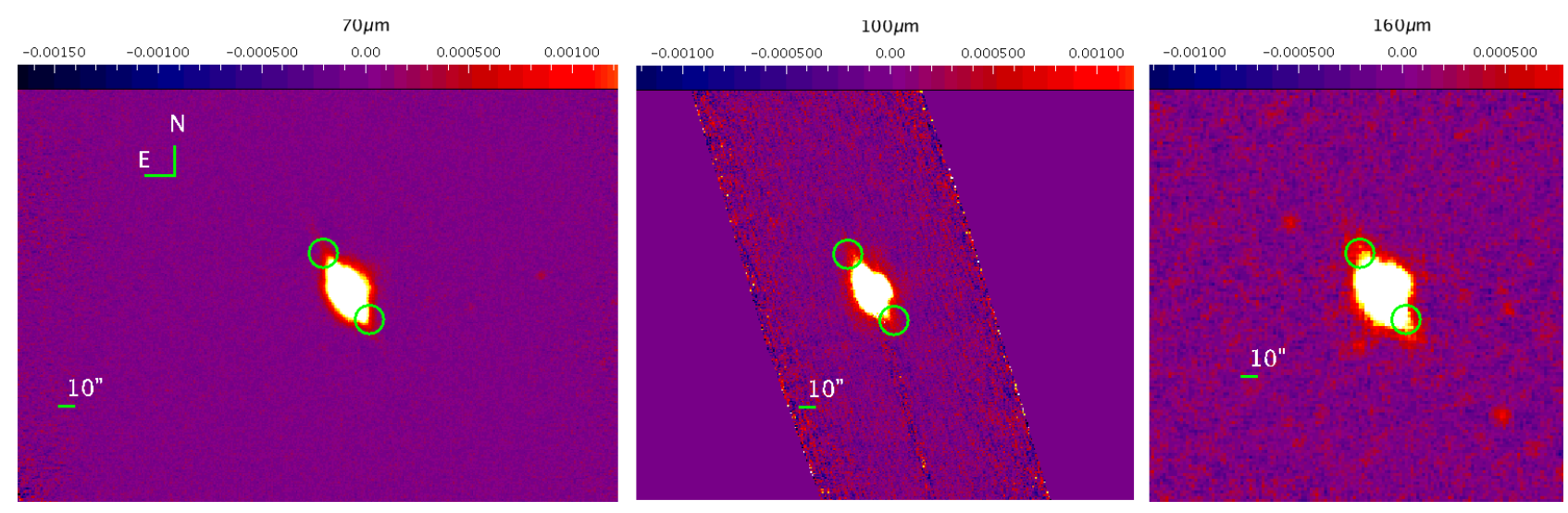

Fig. 3. Surface brightness maps in the PACS bands with the $10^{\prime \prime}$ apertures used to determine an upper limit for the flux of the blob overplotted (left: $70 \mu \mathrm{m}$; middle: $100 \mu \mathrm{m}$; right: $160 \mu \mathrm{m}$ ).

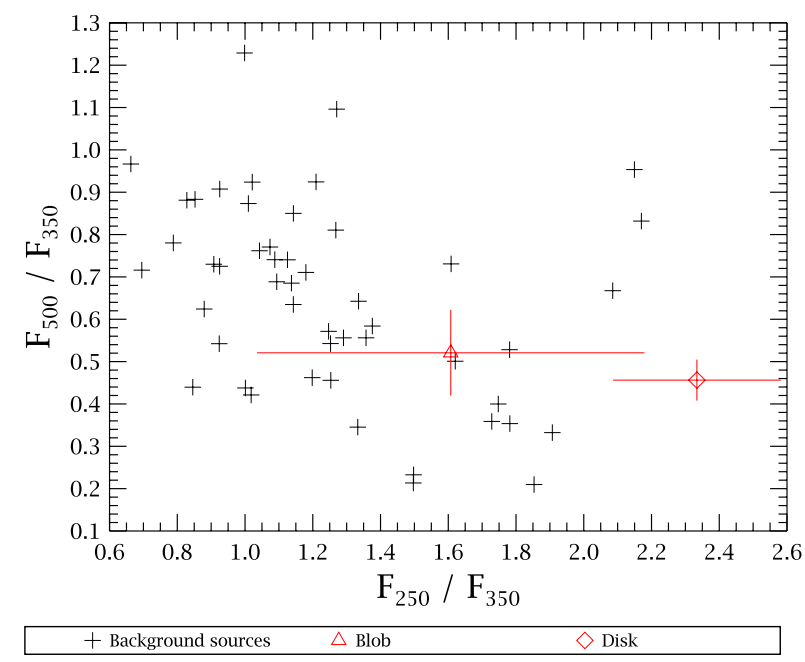

Fig. 4. Colour-colour diagram of the $\beta$ Pic debris disk, the blob and the background sources. The error bars for the fluxes of the background galaxies are not visible on this scale.

Table 5. Median FWHM of the background sources and the FWHM of the blob in the three SPIRE bands.

\begin{tabular}{lcc}
\hline \hline Wavelength $[\mu \mathrm{m}]$ & Background sources ["] & Blob ["] \\
\hline 250 & $18.7 \pm 5.5$ & 23.4 \\
350 & $25.7 \pm 11.2$ & 33.4 \\
500 & $38.2 \pm 19.5$ & 37.2 \\
\hline
\end{tabular}

yielded the FWHM of the blob in the three bands. The results are listed in Table 5.

The blob is not resolved, and its size is clearly consistent with the size of the background sources. This is a second indication that the blob is a background source.

\subsection{Far-infrared SED of the blob and the disk, and interpretation as dust spectrum}

Another test for the hypothesis that the blob is a background source are the relative spectral energy distributions (SEDs) of the disk and the blob. Figure 5 shows the SED of the debris disk with fluxes obtained with Herschel, SCUBA, LABOCA, and SIMBA, normalised to the flux of the disk at $250 \mu \mathrm{m}$, as well as the flux points for the blob, normalised to the flux of the blob at $250 \mu \mathrm{m}$

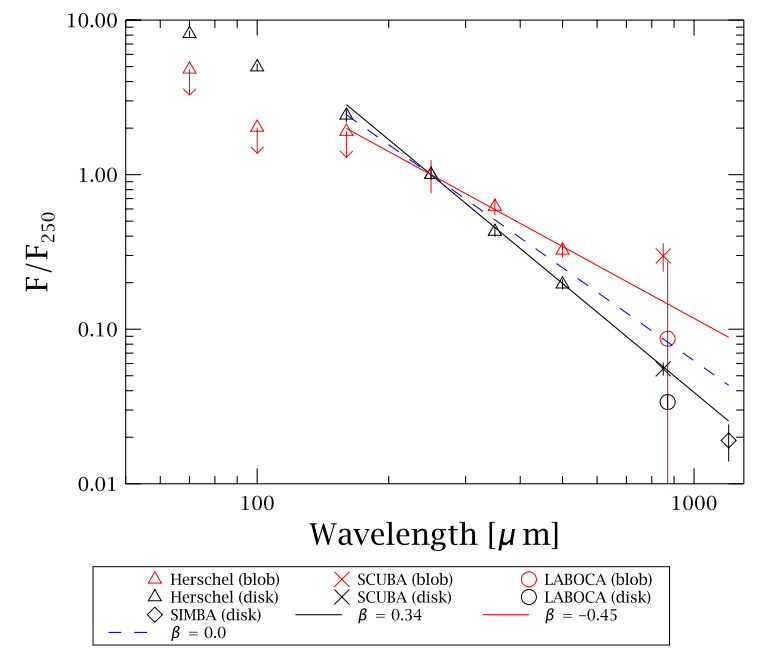

Fig. 5. Spectral energy distribution of the debris disk (in black) and the blob (in red), normalised to their repective flux at $250 \mu \mathrm{m}$. The Herschel fluxes of the disk were obtained by integrating over a target radius of $60^{\prime \prime}$, the SPIRE fluxes of the blob by integrating over a target radius of $20^{\prime \prime}$. The PACS fluxes of the blob are upper limits, determined as described in Sect.3. Additional flux points for the disk were taken from Holland et al. (1998) (SCUBA: $850 \mu \mathrm{m}$ ), Nilsson et al. (2009) (LABOCA: $870 \mu \mathrm{m}$ ) and Liseau et al. (2003) (SIMBA: $1.2 \mathrm{~mm}$ ). The SCUBA and SIMBA fluxes were integrated over a $40^{\prime \prime}$ radius aperture while the LABOCA flux point was obtained by fitting a Gaussian to the disk. Additional flux points for the blob were taken from Dent et al. (2000) (SCUBA: $850 \mu \mathrm{m}$ ) and Nilsson et al. (2009) (LABOCA: $870 \mu \mathrm{m}$ ). Overplotted are three modified Rayleigh-Jeans laws $\left(F_{v} \propto v^{\beta+2}\right)$. The spectral indices have been determined using the fluxes at wavelengths $\lambda \geq 160 \mu \mathrm{m}$ for the disk and $\lambda \geq 250 \mu \mathrm{m}$ for the blob, with the inverse of the errors on the fluxes used as weight. Note the large error bar for the $870 \mu \mathrm{m}$ flux point of the blob. The $\beta=0$ curve is shown for comparison.

(in red). Overplotted are three modified Rayleigh-Jeans laws $\left(F_{v} \propto v^{\beta+2}\right)$. The spectral index $\beta$ describes the mean dust opacity $\left(\kappa \propto \nu^{\beta}\right)$ and is an indicator of the grain size distribution of the dust. The SED of the disk is best described by a spectral index $\beta=0.34$ (implying (sub) $\mu \mathrm{m}$ grains), while a negative spectral index is needed to fit the SED of the blob $(\beta=-0.45$ produces the best result). The latter can only be explained by a steep temperature gradient that cannot occur on the scale the blob would have if it were a structure in the disk (i.e., about $470 \mathrm{AU}$ in diameter). 


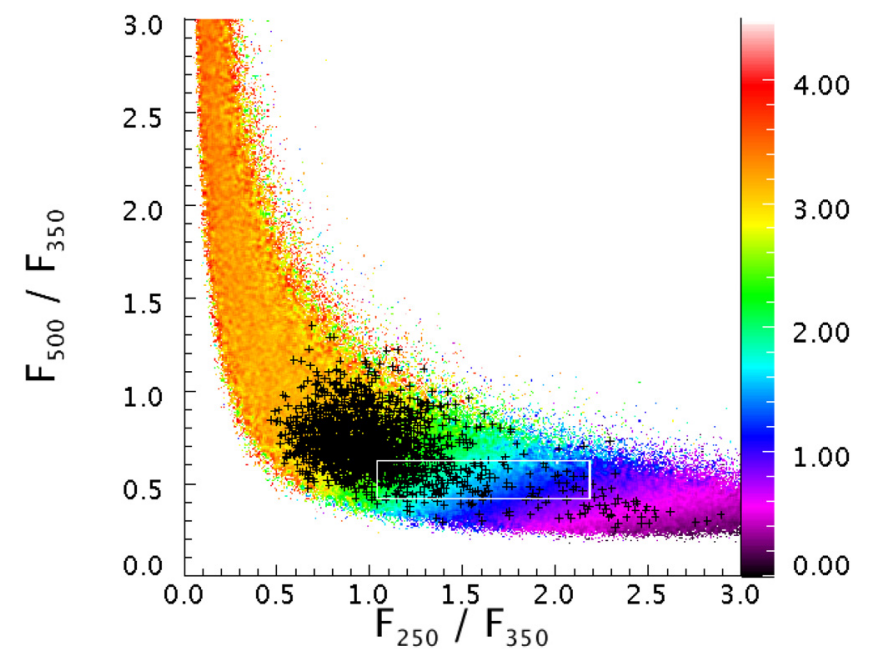

Fig. 6. Colour-colour diagram of the Herschel-ATLAS sources (Amblard et al. 2010). The coloured background indicates the average redshift for $10^{6}$ randomly generated model SEDs. The SPIRE colours of background sources can be overplotted to determine their redshift. Other object types (e.g. the debris disk itself) should not be overplotted. The small crosses are the 1947 galaxies detected in the context of the ATLAS open-time key programme (with a significance greater than $5 \sigma$ at $350 \mu \mathrm{m}$ and $3 \sigma$ at 250 and $500 \mu \mathrm{m}$ ). The overplotted rectangle indicates the position of the blob in this colour-colour space. The redshift in this rectangle lies between 0.78 and 2.6.

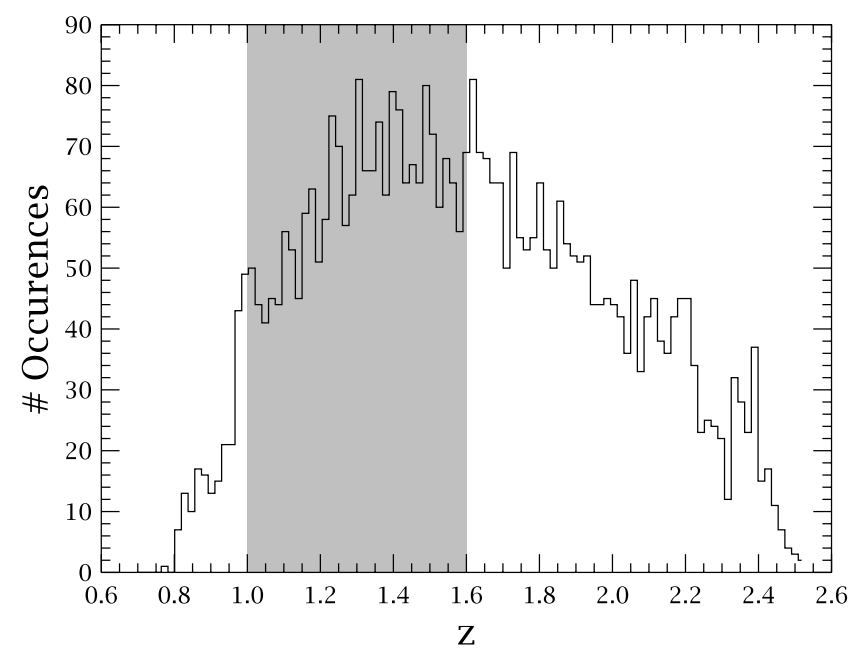

Fig. 7. Histogram of the redshift distribution in the rectangle marked in Fig. 6. Values range between 0.78 and 2.55 . The shaded region indicates the redshift to which we have narrowed down the region.

The submillimetre fluxes of the blob and the disk, as detected by SCUBA, LABOCA, and SIMBA, are summarised in Table B.1.

\subsection{Determination of the redshift}

Assuming the blob really is a background source, we can estimate its redshift based on the far-infrared fluxes. Amblard et al. (2010) investigated the redshift distribution of submillimetre galaxies in the Herschel-ATLAS survey. Their $F_{500} / F_{350}$ vs. $F_{250} / F_{350}$ diagram shows the average redshift in that colour-colour space. In Fig. 6, we have overplotted the datapoint for the blob. Within the error bars, we find redshifts between 0.78 and 2.55 .

Assuming the blob is a background galaxy with redshift $z=1.6$, we derive a luminosity of the order of $10^{26} \mathrm{~W} \mathrm{~Hz}^{-1}$ at $250 \mu \mathrm{m}$ (from the integrated flux at that wavelength). The luminosity distance $d_{\mathrm{L}}$ we used for this calculation was derived from the relationship in Fig. 1 of Choudhury \& Padmanabhan (2005). The luminosity function of Eales et al. (2010, their Fig. 4) shows this is an unlikely high value. Higher redshifts would result in even higher luminosities. Because this is improbable, we estimate $z$ to be no higher than 1.6.

Rejecting the $5 \%$ lowest values for the redshift within the overplotted box (see Fig. 7 for the histogram), we estimate $z$ to be at least 1.0.

\section{Conclusions}

We have investigated the nature of the blob that has been detected to the southwest of the debris disk of $\beta$ Pic not only with Herschel, but also with SCUBA, LABOCA, and SIMBA. By comparing the submillimetre colours of the blob with the colours of the debris disk itself and with the background sources that are present in the images, we conclude that the blob is not a structure in the disk, as previously suggested, but a background galaxy. Support for this hypothesis was found from differences in the spectral indices and SEDs between the disk and the blob, and the fact that the size of the blob is consistent with the size of the background sources. Comparing the colour of the blob to the colour-colour diagram from Amblard et al. (2010) yielded a rough estimate for the redshift of the blob ( $z$ between 0.78 and 2.55), which we narrowed down to $z$ between 1.0 and 1.6.

Ultimately, the nature of the blob could be probed by spectroscopy. The redshift of a galaxy can be determined based on the $\mathrm{CO}$ emission, because the spacing between two successive transitions shrinks with redshift (as $z+1)$. The large total bandwidth and high angular resolution make ALMA the ideal instrument to study the $\mathrm{CO}$ emission of the blob, and determine its redshift. For a normal galaxy like the Milky Way, with $z=3$, the study of the $\mathrm{CO}$ emission takes less than $24 \mathrm{~h}$ observing time (Wootten \& Wilson 2005).

Band 3, the most sensitive available band, will show $\mathrm{CO} 2-1$ transitions for $z=1-2$. Higher (lower) redshifts would move $\mathrm{CO} 2-1$ out of the band, and $\mathrm{CO} 3-2$ (CO 1-0) into the bandpass. Observing additional lines in, e.g., band 7 (with the penalty of lower sensitivity), or JVLA (for CO 1-0) can rule out any ambiguity.

Acknowledgements. The authors thank the referee for constructive comments that have improved the paper in several aspects. This work was supported in part by the Belgian Federal Science Policy Office via the PRODEX Programme of ESA. PACS has been developed by a consortium of institutes led by MPE (Germany) and including UVIE (Austria); KU Leuven, CSL, IMEC (Belgium); CEA, OAMP (France); MPIA (Germany); IFSI, OAP/AOT, OAA/CAISMI, LENS, SISSA (Italy); IAC (Spain). This development has been supported by the funding agencies BMVIT (Austria), ESA PRODEX (Belgium), CEA/CNES (France), DLR (Germany), ASI (Italy), and CICT/MCT (Spain). The authors thank A. Amblard for providing the data to generate Fig. 6.

\section{Appendix A: Photometry of the background galaxies in the SPIRE wavelength bands}

Table A.1 summarises the positions and photometry in the three SPIRE bands for the extracted background galaxies. 
A\&A 541, A3 (2012)

Table A.1. Positions and photometry of the background sources in the SPIRE wavelength bands.

\begin{tabular}{|c|c|c|c|c|c|}
\hline Index & $\alpha[\mathrm{h} \mathrm{m} \mathrm{s}]$ & $\delta\left[{ }^{\circ}{ }^{\prime \prime \prime}\right]$ & $F_{250}[\mathrm{mJy}]$ & $F_{350}[\mathrm{mJy}]$ & $F_{500}[\mathrm{mJy}]$ \\
\hline 1 & $\begin{array}{lll}5 & 47 & 02.97\end{array}$ & $\begin{array}{lll}-51 & 03 & 46.27\end{array}$ & 86.55 & 53.41 & 26.75 \\
\hline 2 & $\begin{array}{lll}5 & 47 & 14.96\end{array}$ & $\begin{array}{lll}-51 & 08 & 11.93\end{array}$ & 88.56 & 84.93 & 64.70 \\
\hline 3 & $\begin{array}{lll}5 & 47 & 08.37\end{array}$ & $\begin{array}{lll}-51 & 05 & 21.58\end{array}$ & 66.35 & 61.80 & 47.62 \\
\hline 4 & $547 \quad 41.45$ & $\begin{array}{lll}-51 & 08 & 31.18\end{array}$ & 49.83 & 27.97 & 09.89 \\
\hline 5 & $\begin{array}{lll}5 & 47 & 10.16\end{array}$ & $\begin{array}{lll}-51 & 07 & 52.80\end{array}$ & 51.50 & 47.34 & 35.06 \\
\hline 6 & $\begin{array}{lll}5 & 47 & 29.32\end{array}$ & $\begin{array}{lll}-51 & 07 & 03.24\end{array}$ & 44.35 & 39.41 & 29.18 \\
\hline 7 & $\begin{array}{lll}5 & 47 & 19.58\end{array}$ & $\begin{array}{lll}-51 & 01 & 25.95\end{array}$ & 44.32 & 47.92 & 34.75 \\
\hline 8 & $\begin{array}{lll}5 & 47 & 03.42\end{array}$ & $\begin{array}{lll}-51 & 06 & 00.39\end{array}$ & 40.79 & 30.55 & 19.63 \\
\hline 9 & $\begin{array}{lll}5 & 47 & 10.92\end{array}$ & $\begin{array}{lll}-51 & 03 & 52.90\end{array}$ & 36.46 & 39.41 & 35.76 \\
\hline 10 & $\begin{array}{lll}5 & 46 & 46.97\end{array}$ & $\begin{array}{lll}-51 & 08 & 54.68\end{array}$ & 56.67 & 42.52 & 14.68 \\
\hline 11 & $\begin{array}{lll}5 & 47 & 05.71\end{array}$ & $\begin{array}{lll}-51 & 05 & 47.38\end{array}$ & 36.85 & 30.46 & 28.16 \\
\hline 12 & $\begin{array}{lll}5 & 47 & 01.50\end{array}$ & $\begin{array}{lll}-51 & 03 & 11.85\end{array}$ & 36.67 & 40.39 & 29.48 \\
\hline 13 & $\begin{array}{lll}5 & 47 & 24.11\end{array}$ & $\begin{array}{lll}-51 & 03 & 07.39\end{array}$ & 38.46 & 25.69 & 05.48 \\
\hline 14 & $\begin{array}{lll}5 & 47 & 11.78\end{array}$ & $\begin{array}{lll}-51 & 04 & 42.97\end{array}$ & 31.45 & 31.49 & 38.70 \\
\hline 15 & $\begin{array}{lll}5 & 47 & 08.21\end{array}$ & $\begin{array}{lll}-51 & 04 & 07.02\end{array}$ & 32.21 & 25.35 & 27.79 \\
\hline 16 & $\begin{array}{lll}5 & 47 & 00.32\end{array}$ & $\begin{array}{lll}-50 & 59 & 00.80\end{array}$ & 32.99 & 29.02 & 19.88 \\
\hline 17 & $\begin{array}{lll}5 & 47 & 06.40\end{array}$ & $\begin{array}{lll}-51 & 00 & 46.39\end{array}$ & 34.05 & 24.75 & 14.45 \\
\hline 18 & $\begin{array}{lll}5 & 47 & 05.10\end{array}$ & $\begin{array}{lll}-51 & 04 & 24.99\end{array}$ & 31.98 & 29.24 & 20.13 \\
\hline 19 & $\begin{array}{lll}5 & 46 & 51.74\end{array}$ & $\begin{array}{lll}-51 & 01 & 18.91\end{array}$ & 32.19 & 32.15 & 14.07 \\
\hline 20 & $\begin{array}{lll}5 & 47 & 24.20\end{array}$ & $\begin{array}{lll}-51 & 01 & 12.17\end{array}$ & 31.97 & 18.50 & 06.63 \\
\hline 21 & $\begin{array}{lll}5 & 47 & 26.50\end{array}$ & $\begin{array}{lll}-51 & 08 & 47.06\end{array}$ & 34.41 & 16.50 & 11.01 \\
\hline 22 & $\begin{array}{lll}5 & 46 & 53.13\end{array}$ & $\begin{array}{lll}-51 & 02 & 39.18\end{array}$ & 27.32 & 14.74 & 03.09 \\
\hline 23 & $\begin{array}{lll}5 & 47 & 46.42\end{array}$ & $\begin{array}{lll}-51 & 03 & 22.98\end{array}$ & 28.83 & 23.02 & 12.49 \\
\hline 24 & $\begin{array}{lll}5 & 47 & 44.64\end{array}$ & $\begin{array}{lll}-50 & 59 & 59.36\end{array}$ & 29.00 & 15.20 & 05.05 \\
\hline 25 & $\begin{array}{lll}5 & 46 & 58.97\end{array}$ & $\begin{array}{lll}-51 & 03 & 37.51\end{array}$ & 25.57 & 22.38 & 19.02 \\
\hline 26 & $\begin{array}{lll}5 & 46 & 55.88\end{array}$ & $\begin{array}{lll}-50 & 59 & 19.69\end{array}$ & 26.61 & 26.05 & 24.07 \\
\hline 27 & $\begin{array}{lll}5 & 47 & 28.34\end{array}$ & $\begin{array}{lll}-51 & 00 & 31.02\end{array}$ & 24.14 & 27.45 & 17.13 \\
\hline 28 & $\begin{array}{lll}5 & 47 & 11.41\end{array}$ & $\begin{array}{lll}-51 & 07 & 22.61\end{array}$ & 26.36 & 31.83 & 28.04 \\
\hline 29 & $\begin{array}{lll}5 & 47 & 24.42\end{array}$ & $\begin{array}{lll}-51 & 08 & 53.03\end{array}$ & 23.56 & 18.58 & 15.06 \\
\hline 30 & $\begin{array}{lll}5 & 47 & 03.81\end{array}$ & $\begin{array}{lll}-51 & 01 & 38.75\end{array}$ & 23.51 & 18.86 & 10.78 \\
\hline 31 & $\begin{array}{lll}5 & 47 & 01.02\end{array}$ & $\begin{array}{lll}-51 & 00 & 24.24\end{array}$ & 22.22 & 12.47 & 06.59 \\
\hline 32 & $\begin{array}{lll}5 & 47 & 28.00\end{array}$ & $\begin{array}{lll}-51 & 07 & 44.27\end{array}$ & 21.76 & 19.04 & 12.09 \\
\hline 33 & 54654.81 & $\begin{array}{lll}-51 & 04 & 45.89\end{array}$ & 20.21 & 29.07 & 20.81 \\
\hline 34 & $\begin{array}{lll}5 & 47 & 46.63\end{array}$ & $\begin{array}{lll}-51 & 02 & 50.88\end{array}$ & 18.67 & 15.82 & 11.24 \\
\hline 35 & $\begin{array}{lll}5 & 47 & 28.09\end{array}$ & $\begin{array}{lll}-51 & 01 & 24.72\end{array}$ & 19.02 & 15.18 & 06.92 \\
\hline 36 & $\begin{array}{lll}5 & 47 & 48.58\end{array}$ & $\begin{array}{lll}-51 & 04 & 52.28\end{array}$ & 19.67 & 12.23 & 08.94 \\
\hline 37 & $\begin{array}{lll}5 & 47 & 10.79\end{array}$ & $\begin{array}{lll}-51 & 02 & 04.77\end{array}$ & 16.18 & 13.50 & 06.24 \\
\hline 38 & $\begin{array}{lll}5 & 47 & 03.83\end{array}$ & $\begin{array}{lll}-51 & 00 & 16.54\end{array}$ & 18.47 & 18.14 & 07.64 \\
\hline 39 & $\begin{array}{lll}5 & 47 & 45.58\end{array}$ & $\begin{array}{lll}-51 & 04 & 52.72\end{array}$ & 16.10 & 07.42 & 06.17 \\
\hline 40 & $\begin{array}{lll}5 & 47 & 22.00\end{array}$ & $\begin{array}{lll}-51 & 05 & 27.15\end{array}$ & 13.79 & 09.21 & 02.14 \\
\hline 41 & $\begin{array}{lll}5 & 47 & 22.66\end{array}$ & $\begin{array}{lll}-51 & 07 & 32.10\end{array}$ & 14.72 & 08.42 & 03.36 \\
\hline 42 & $\begin{array}{lll}5 & 47 & 09.78\end{array}$ & $-5100 \quad 11.45$ & 12.65 & 09.32 & 05.18 \\
\hline 43 & $\begin{array}{lll}5 & 47 & 13.95\end{array}$ & $-50 \quad 58 \quad 56.53$ & 13.08 & 06.08 & 05.80 \\
\hline 44 & $5 \quad 4650.31$ & -505959.64 & 14.08 & 17.86 & 13.94 \\
\hline 45 & $\begin{array}{lll}54730.64\end{array}$ & $\begin{array}{lll}-51 & 08 & 37.85\end{array}$ & 10.32 & 12.11 & 10.70 \\
\hline 46 & $\begin{array}{lll}5 & 47 & 03.08\end{array}$ & $\begin{array}{lll}-51 & 05 & 19.24\end{array}$ & 10.16 & 10.06 & 08.78 \\
\hline 47 & 54652.54 & -505956.15 & 11.36 & 17.14 & 16.57 \\
\hline 48 & 54657.46 & $\begin{array}{lll}-51 & 08 & 35.38\end{array}$ & 10.75 & 12.72 & 05.59 \\
\hline 49 & $\begin{array}{lll}5 & 47 & 07.62\end{array}$ & $\begin{array}{lll}-51 & 01 & 52.54\end{array}$ & 10.11 & 07.83 & 04.35 \\
\hline 50 & $\begin{array}{lll}5 & 46 & 55.04\end{array}$ & $\begin{array}{lll}-51 & 08 & 30.49\end{array}$ & 10.55 & 11.42 & 06.19 \\
\hline
\end{tabular}


Table B.1. Submillimetre fluxes of the disk and the blob as detected by SCUBA, LABOCA, and SIMBA. The blob was not observed at $1.2 \mathrm{~mm}$.

\begin{tabular}{lccc}
\hline \hline Wavelength $[\mu \mathrm{m}]$ & Flux disk $[\mathrm{mJy}]$ & Flux blob $[\mathrm{mJy}]$ & References \\
\hline 850 & $104.3 \pm 10.0$ & $24 \pm 5$ & 1,2 \\
870 & $63.6 \pm 6.7$ & $7.0 \pm 14.9$ & 3 \\
1200 & $35.9 \pm 9.7$ & $/$ & 4 \\
\hline
\end{tabular}

References. (1) Holland et al. (1998); (2) Dent et al. (2000); (3) Nilsson et al. (2009); (4) Liseau et al. (2003).

\section{Appendix B: Submillimetre fluxes of the disk and blob}

In Table B. 1 the submillimetre fluxes of the disk and the blob as measured with SCUBA, LABOCA, and SIMBA are listed.

\section{References}

Amblard, A., Cooray, A., Serra, P., et al. 2010, A\&A, 518, L9

Aumann, H. H., Beichman, C. A., Gillett, F. C., et al. 1984, ApJ, 278, L23

Choudhury, T. R., \& Padmanabhan, T. 2005, A\&A, 429, 807

Dent, W. R. F., Walker, H. J., Holland, W. S., \& Greaves, J. S. 2000, MNRAS, 314,702

Eales, S. A., Raymond, G., Roseboom, I. G., et al. 2010, A\&A, 518, L23

Griffin, M. J., Abergel, A., Abreu, A., et al. 2010, A\&A, 518, L3

Heap, S. R., Lindler, D. J., Lanz, T. M., et al. 2000, ApJ, 539, 435

Holland, W. S., Greaves, J. S., Zuckerman, B., et al. 1998, Nature, 392, 788
Kalas, P., \& Jewitt, D. 1995, AJ, 110, 794

Lagrange, A.-M., Bonnefoy, M., Chauvin, G., et al. 2010, Science, 329, 133

Larwood, J. D., \& Kalas, P. G. 2001, MNRAS, 323, 402

Liseau, R., Brandeker, A., Fridlund, M., et al. 2003, A\&A, 402, 183

Mouillet, D., Larwood, J. D., Papaloizou, J. C. B., \& Lagrange, A. M. 1997, MNRAS, 292, 896

Nilsson, R., Liseau, R., Brandeker, A., et al. 2009, A\&A, 508, 1057

Ott, S., \& Science Ground Segment Consortium 2010, in AAS Meeting Abstracts, 216, 413.10

Pantin, E., Lagage, P. O., \& Artymowicz, P. 1997, A\&A, 327, 1123

Pilbratt, G. L., Riedinger, J. R., Passvogel, T., et al. 2010, A\&A, 518, L1

Poglitsch, A., Waelkens, C., Geis, N., et al. 2010, A\&A, 518, L2

Swinyard, B. M., Ade, P., Baluteau, J., et al. 2010, A\&A, 518, L4

Telesco, C. M., Fisher, R. S., Wyatt, M. C., et al. 2005, Nature, 433, 133

van Leeuwen, F. 2007, A\&A, 474, 653

Vandenbussche, B., Sibthorpe, B., Acke, B., et al. 2010, A\&A, 518, L133

Wootten, A., \& Wilson, T. 2005, ALMA Scientific Specifications and Requirements 